\title{
Ultrafast photoinduced relaxation dynamics of the indoline dye D149 in organic solvents $\dagger$
}

\author{
Peter W. Lohse, ${ }^{a}$ Julia Kuhnt, ${ }^{b}$ Sergey I. Druzhinin, ${ }^{a}$ Mirko Scholz, ${ }^{b}$ \\ Maria Ekimova, ${ }^{a}$ Torsten Oekermann, ${ }^{c}$ Thomas Lenzer ${ }^{* a}$ and Kawon Oum ${ }^{a}$
}

Received 27th July 2011, Accepted 21st September 2011

DOI: $10.1039 / \mathrm{clcp22429h}$

The relaxation dynamics of the indoline dye D149, a well-known sensitizer for photoelectrochemical solar cells, have been extensively characterized in various organic solvents by combining results from ultrafast pump-supercontinuum probe (PSCP) spectroscopy, transient UV-pump VIS-probe spectroscopy, time-correlated single-photon counting (TCSPC) measurements as well as steady-state absorption and fluorescence. In the steady-state spectra, the position of the absorption maximum shows only a weak solvent dependence, whereas the fluorescence Stokes shift $\Delta \tilde{\nu}_{\mathrm{F}}$ correlates with solvent polarity. Photoexcitation at around $480 \mathrm{~nm}$ provides access to the $S_{1}$ state of D149 which exhibits solvation dynamics on characteristic timescales, as monitored by a red-shift of the stimulated emission and spectral development of the excited-state absorption in the transient PSCP spectra. In all cases, the spectral dynamics can be modeled by a global kinetic analysis using a time-dependent $S_{1}$ spectrum. The lifetime $\tau_{1}$ of the $S_{1}$ state roughly correlates with polarity [acetonitrile $(280 \mathrm{ps})<$ acetone $(540 \mathrm{ps})<$ THF $(720 \mathrm{ps})<$ chloroform $(800 \mathrm{ps})$ ], yet in alcohols it is much shorter [methanol $(99 \mathrm{ps})<$ ethanol $(178 \mathrm{ps})<$ acetonitrile $(280 \mathrm{ps})$ ], suggesting an appreciable influence of hydrogen bonding on the dynamics. A minor component with a characteristic time constant in the range 19-30 ps, readily observed in the PSCP spectra of D149 in acetonitrile and THF, is likely due to removal of vibrational excess energy from the $S_{1}$ state by collisions with solvent molecules. Additional weak fluorescence in the range 390-500 $\mathrm{nm}$ is observed upon excitation in the $\mathrm{S}_{0} \rightarrow \mathrm{S}_{2}$ band, which contains short-lived $\mathrm{S}_{2} \rightarrow \mathrm{S}_{0}$ emission of D149. Transient absorption signals after excitation at $377.5 \mathrm{~nm}$ yield an additional time constant in the subpicosecond range, representing the lifetime of the $\mathrm{S}_{2}$ state. $\mathrm{S}_{2}$ excitation also produces photoproducts.

\section{Introduction}

Indolines have emerged as an important class of metal-free sensitizer dyes for photoelectrochemical solar cells, in which they replace standard ruthenium-based complexes, which are more expensive and also have absorption coefficients which are lower by a factor of five. ${ }^{1-9}$ They have been proven to be especially useful in $\mathrm{ZnO}$-based dye-sensitized solar cells, where the typical ruthenium complexes developed for the sensitization

${ }^{a}$ Universität Siegen, Physikalische Chemie, Adolf-Reichwein-Str. 2, 57076 Siegen, Germany.E-mail: lenzer@chemie.uni-siegen.de; Fax: + 49271740 2805; Tel: + 492717402803

${ }^{b}$ Georg-August-Universität Göttingen, Institut für Physikalische Chemie, Tammannstr. 6, 37077 Göttingen, Germany

${ }^{c}$ Institut für Physikalische Chemie und Elektrochemie, Leibniz-Universität Hannover, 30167 Hannover, Germany

$\dagger$ Electronic supporting information (ESI) available: Results of photostability experiments using sub-400 nm excitation in the $\mathrm{S}_{0} \rightarrow \mathrm{S}_{2}$ band of D149 in ethanol solution by cw lamp illumination. See DOI: $10.1039 / \mathrm{clcp} 22429 \mathrm{~h}$ of $\mathrm{TiO}_{2}$ cannot be used, since they tend to etch the $\mathrm{ZnO}$ surface and form aggregates on it. ${ }^{10-13}$ To achieve a better overlap with the solar spectrum, a range of structurally modified indoline dyes with an increasing red-shift of the absorption spectrum have been synthesized. ${ }^{1-3}$ One of the most frequently used indolines for solar-cell applications is D149, which is depicted in Fig. 1. Surprisingly, so far there has been only very limited information on indoline dynamics after photoexcitation, e.g., a study of Fakis et al. for D149 in toluene and acetonitrile and on $\mathrm{Al}_{2} \mathrm{O}_{3}$ and $\mathrm{TiO}_{2}$ surfaces using single-wavelength fluorescence up-conversion, ${ }^{14}$ which will be discussed with respect to our current findings below. In addition, DFT/TD-DFT calculations have been reported and assigned considerable charge transfer (CT) character to the $\mathrm{S}_{1}$ state. $^{15,16}$

Here we present a comprehensive investigation of D149 in various organic solvents using ultrafast transient absorption spectroscopy, time-correlated single-photon counting (TCSPC) measurements as well as steady-state absorption and 


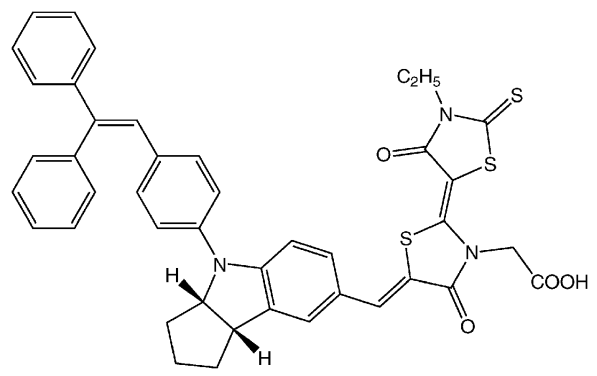

Fig. 1 Chemical structure of indoline D149 (adapted from ref. 6).

fluorescence. After $S_{1}$ excitation, the photoinduced dynamics of this sensitizer dye appear to be fairly simple, despite its rather complicated structure. In contrast, the dynamics after $\mathrm{S}_{2}$ excitation turn out to be much more complex and point toward the additional formation of photoproducts.

\section{Experimental}

\subsection{Pump-supercontinuum probe (PSCP) spectroscopy}

A detailed description of PSCP spectroscopy was already given elsewhere ${ }^{17}$ and here we only briefly discuss the setup used in our experiments: ${ }^{18-20}$ a regenerative Ti:Sa system (Spectra-Physics Hurricane, center wavelength: $780 \mathrm{~nm}$, repetition rate: $920 \mathrm{~Hz}$, pulse length: $100 \mathrm{fs}, 1 \mathrm{~mJ}$ pulse $^{-1}$ ) was employed for pumping two home-built NOPAs. ${ }^{21,22}$ The pump NOPA generated pulses centered at 476-484 nm, which were compressed by a pair of quartz prisms and then attenuated by pellicle beam splitters to excite D149 via its $\mathrm{S}_{0} \rightarrow \mathrm{S}_{1}$ transition (photon density ca. $5 \times 10^{14} \mathrm{~cm}^{-2}$ ). The second NOPA generated a wavelength of $c a .550 \mathrm{~nm}$ which was also compressed by a quartz prism pair and then focused into a $1 \mathrm{~mm}$ thick calcium fluoride plate generating a multifilament supercontinuum, where the range $340-770 \mathrm{~nm}$ was used in the current study. The supercontinuum was spectrally filtered by a dye solution and then split up into reference and sample beams. Pump and probe pulses were overlapped at magicangle polarization in a stainless steel flow cell with $400 \mu \mathrm{m}$ path length and $200 \mu \mathrm{m}$ thick quartz windows. Reference and sample spectra were dispersed by two spectrographs with 512 element photodiode arrays. Transient spectra for each pump-probe delay represent the average of three independent scans, each consisting of 500 laser shots employing single-shot baseline correction. Solvent signals arising during the crosscorrelation time were not subtracted from the experiment signals, and can be easily discerned by their characteristic time dependence. The pump-probe intensity cross-correlation time in the current experiments was 90-100 fs and the time accuracy $10 \mathrm{fs}$.

\subsection{UV pump-VIS probe transient absorption spectroscopy}

Details of this setup can be found in previous publications, ${ }^{23,24}$ and here we focus only on the specifics relevant for the current experiments: the output of a mode-locked Ti:sapphire oscillator (Spectra-Physics Tsunami, $80 \mathrm{MHz}$; pump source: SpectraPhysics Millenia Xs Nd: $\mathrm{YVO}_{4}$ laser) generated red pulses at $755 \mathrm{~nm}$ with an average power of $1.2 \mathrm{~W}$. The laser beam was split into two parts. One beam traversed an acousto-optic modulator (AOM, $2 \mathrm{MHz}$ modulation frequency) with subsequent frequency-doubling in an LBO crystal, resulting in an intensity-modulated UV pump beam $(377.5 \mathrm{~nm}$, pulse energy $<0.1 \mathrm{~nJ})$. The other part had a typical energy $<1 \mathrm{~nJ}$ pulse $\mathrm{e}^{-1}$ and was directly used as a probe beam $(755 \mathrm{~nm})$. The time resolution of the setup was $c a$. 130 fs. Pump and probe pulses at magic-angle polarization were collinearly recombined and focused into a quartz flow cuvette containing D149 in the solvent of interest. Transient absorption was detected by an avalanche photodiode using an appropriate set of cut-off filters for the pump beam wavelength. The signal was processed by a lock-in amplifier which used the same $2 \mathrm{MHz}$ reference as the AOM.

\subsection{Nanosecond time-correlated single photon counting (TCSPC)}

The basic setup of the TCSPC system was described before. ${ }^{25,26}$ In the current measurements, a solution of D149 in THF, acetonitrile or chloroform (OD 0.3) was excited by a pulsed $\mathrm{N}_{2}$ flash lamp (FWHM $2 \mathrm{~ns}$ ) at a repetition rate of $50 \mathrm{kHz}$. The unpolarized radiation of the $\mathrm{N}_{2}$ line at $337 \mathrm{~nm}$ was selected by a monochromator (Jobin-Yvon H.20 UV, bandwidth: 1-2 nm) and emission was detected at the maximum of the fluorescence spectrum using an identical monochromator combined with a photomultiplier (Philips XP2020). The signal was fed into a timeto-amplitude converter (Tennelec TC 862) connected to a multichannel analyzer (Silena ADC 7423 UHS). A scatter solution (Ludox, Du Pont) and the D149 sample solution were alternately translated into the excitation beam until 1000 counts in the maximum were accumulated. Decay curves were deconvolved with the instrument response function (IRF) resulting in monoexponential decays. With this setup, single exponential decay time constants down to a few hundred ps can be determined.

\subsection{Steady-state absorption and fluorescence}

Absorption spectra were recorded on Varian Cary 5E and 5000 spectrometers with baseline correction. Fluorescence spectra were detected using Horiba Jobin-Yvon Fluorolog-3 and Varian Cary Eclipse spectrometers. Fluorescence raw data were corrected for the instrument response function.

\subsection{Photostability experiments in the UV}

For photostability measurements in the $\mathrm{S}_{0} \rightarrow \mathrm{S}_{2}$ band of D149 below $400 \mathrm{~nm}$, the light from a cw high-pressure $\mathrm{Hg}-\mathrm{Xe}$ lamp (Osram HBO, $200 \mathrm{~W}$ ) was passed through an appropriate set of optical glass filters (Schott UG1 and WG320) and mildly focused into the sample cuvette $(10 \mathrm{~mm} \times 10 \mathrm{~mm})$ by a quartz lens $(f=150 \mathrm{~mm})$ at an angle of $90^{\circ}$ with respect to the beam path of the absorption spectrometer. The resulting power density at the sample was $c a .50 \mathrm{~mW} \mathrm{~cm}{ }^{-2}$.

\subsection{Chemicals}

The indoline dye D149 was purchased from Inabata UK Ltd. and used without further purification. Solvents had a specified purity of $99 \%$ or better. The absorption, fluorescence and TCSPC measurements were carried out at $298 \mathrm{~K}$ in nitrogen saturated solution. We note that solutions of D149 in acetonitrile showed absorption shifts in the $\mathrm{nm}$ range and sometimes formation of a weak blue shoulder, which are tentatively 
assigned to a side-reaction of $\mathrm{D} 149$, possibly with $\mathrm{O}_{2}$ or aggregate formation.

\section{Results and discussion}

\subsection{Steady-state absorption and fluorescence of D149}

Steady-state absorption and fluorescence spectra in six organic solvents are depicted in Fig. 2. Characteristic values are summarized in Table 1 including the polarizability $R(n)=\left(n^{2}-1\right) /\left(n^{2}+2\right)$ and the polarity function $\Delta f=R(\varepsilon)-R(n)=(\varepsilon-1) /(\varepsilon+2)-\left(n^{2}-1\right) /\left(n^{2}+2\right)$, calculated from tabulated $n$ and $\varepsilon$ values. ${ }^{27}$ The absorption spectra show only a weak solvent dependence. They consist of two bands centered at $530 \mathrm{~nm}$ and $390 \mathrm{~nm}$, which correspond to the $\mathrm{S}_{0} \rightarrow \mathrm{S}_{1}$ and $\mathrm{S}_{0} \rightarrow \mathrm{S}_{2}$ transitions, respectively (slightly shifted in chloroform).

The fluorescence spectra were obtained after excitation at the maximum of the $\mathrm{S}_{0} \rightarrow \mathrm{S}_{1}$ absorption band. They exhibit a substantial Stokes shift $\Delta \tilde{\nu}_{\mathrm{F}}$, which is correlated with solvent polarity (e.g. $2780 \mathrm{~cm}^{-1}$ in chloroform compared to $4050 \mathrm{~cm}^{-1}$ in acetonitrile), indicating that the dipole moment of D149

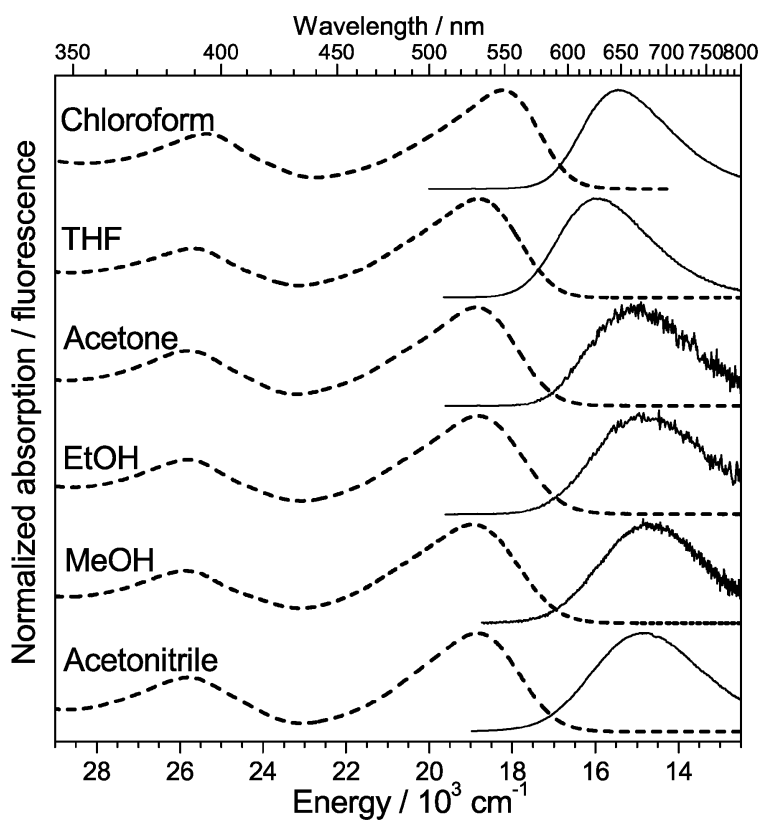

Fig. 2 Absorption spectra (dashed lines) and fluorescence spectra (solid lines) of D149 in different organic solvents. Fluorescence spectra were recorded after excitation at the absorption maximum of the $\mathrm{S}_{0} \rightarrow \mathrm{S}_{1}$ band in each case. increases upon photoexcitation, see also Table 1. We note that Fakis et al. ${ }^{14}$ reported the fluorescence maximum in acetonitrile at $c a .615 \mathrm{~nm}$, which is blue-shifted by $49 \mathrm{~nm}$ with respect to our value. This large difference is likely due to a missing correction of their fluorescence spectrum with respect to detector sensitivity. Similarly, a previous study of Le Bahers et al. ${ }^{16}$ reports the $S_{1}$ emission maximum in methanol at $644 \mathrm{~nm}$ compared to the $670 \mathrm{~nm}$ found in our study. The difference of $26 \mathrm{~nm}$ and the additional shoulder on the red side of their fluorescence spectra also suggest a missing correction for detector sensitivity in that study. A similar explanation might hold for chloroform, where Dentani et al. find the fluorescence maximum at $636 \mathrm{~nm},{ }^{8}$ whereas our value is $643 \mathrm{~nm}$.

Fig. 3(A) contains $S_{1} \rightarrow S_{0}$ steady-state fluorescence spectra of D149 in acetonitrile and THF recorded after $\mathrm{S}_{0} \rightarrow \mathrm{S}_{1}$ excitation at 487 and $522 \mathrm{~nm}$ (dotted lines), respectively, and $\mathrm{S}_{0} \rightarrow \mathrm{S}_{2}$ excitation at $380 \mathrm{~nm}$ (solid lines) for D149 in both solvents. After $\mathrm{S}_{2}$ excitation, we observe a slight red-shift of 130 and $50 \mathrm{~cm}^{-1}$ for the fluorescence in acetonitrile and THF, respectively. Possible reasons for this discrepancy will be discussed in Section 3.4. As can be seen from Fig. 3(B), the fluorescence spectra after $\mathrm{S}_{2}$ excitation show an additional weak pedestal which starts to rise around $26000 \mathrm{~cm}^{-1}$. It is rather flat and merges with the $S_{1}$ emission. We note that this result is in contrast to Fakis et al., who observed two distinct emission bands with a dip at $c a .530 \mathrm{~nm}$, which were assigned to separate $S_{1}$ and $S_{2}$ emissions. ${ }^{14}$ The pedestals in Fig. 3(B) can be assigned to weak $\mathrm{S}_{2} \rightarrow \mathrm{S}_{0}$ emission, but contributions from an impurity are probably dominant (see Section 3.4). We note that the $\mathrm{S}_{2} \rightarrow \mathrm{S}_{0} / \mathrm{S}_{1} \rightarrow \mathrm{S}_{0}$ emission band ratio, which we obtained after deconvolution, is much smaller than in the spectra reported by Fakis et al., ${ }^{14}$ which also suggest a missing correction for detector sensitivity in their experiments. The extremely weak $\mathrm{S}_{2} \rightarrow \mathrm{S}_{0}$ emission already points toward a very short lifetime of the $\mathrm{S}_{2}$ state. Note also that the $\mathrm{S}_{2} \rightarrow \mathrm{S}_{0} / \mathrm{S}_{1} \rightarrow \mathrm{S}_{0}$ emission band ratio in acetonitrile is larger than in THF. We will discuss the resulting implications for the $S_{2} / S_{1}$ lifetime ratio in more detail in Section 3.4.

\subsection{Transient PSCP spectra and TCSPC experiments}

Ultrafast transient broadband absorption spectra $\Delta \mathrm{OD}(t)$ of D149 in acetonitrile, methanol and THF are shown in Fig. 4-6, respectively. D149 was excited to the $S_{1}$ state at $476 \mathrm{~nm}$ (acetonitrile), $479 \mathrm{~nm}$ (methanol) and $484 \mathrm{~nm}$ (THF). Table 2 contains a summary of experimental conditions and kinetic information extracted from the PSCP spectra, including

Table 1 Characteristics of solvent dependent D149 steady-state absorption and fluorescence spectra $\left(\mathrm{S}_{0} \rightarrow \mathrm{S}_{1}\right.$ transition)

\begin{tabular}{llllllll}
\hline Solvent & $R(n)^{a}$ & $\Delta f^{a}$ & $\lambda_{\text {max. }}^{\text {Abs. }} / \mathrm{nm}$ & $\tilde{\nu}_{\text {max. }}^{\text {Abs. }} / \mathrm{cm}^{-1}$ & $\lambda_{\text {max. }}^{\text {Fl. }} / \mathrm{nm}$ & $\tilde{\nu}_{\text {max. }}^{\text {Fl. }} / \mathrm{cm}^{-1}$ & $\Delta \tilde{\nu}_{\mathrm{F}}=\left(\tilde{\nu}_{\text {max. }}^{\text {Abs. }}-\tilde{\nu}_{\text {max. }}^{\text {Fl. }}\right) / \mathrm{cm}^{-1}$ \\
\hline Acetonitrile & 0.212 & 0.711 & 529 & 18920 & 664 & 14870 & 4050 \\
Methanol & 0.203 & 0.711 & 528 & 18950 & 670 & 14690 & 4260 \\
Ethanol & 0.221 & 0.669 & 531 & 18820 & 664 & 14800 & 4020 \\
Acetone & 0.218 & 0.651 & 530 & 18870 & 659 & 15010 & 3860 \\
THF & 0.246 & 0.439 & 531 & 18830 & 621 & 15960 & 2870 \\
Chloroform & 0.267 & 0.293 & 549 & 18230 & 643 & 15450 & 2780
\end{tabular}

${ }^{a} \Delta f=R(\varepsilon)-R(n)$, where $R(\varepsilon)=(\varepsilon-1) /(\varepsilon+2)$ and $R(n)=\left(n^{2}-1\right) /\left(n^{2}+2\right)$ with the dielectric constant $\varepsilon$ and the index of refraction $n$ of the solvent. 


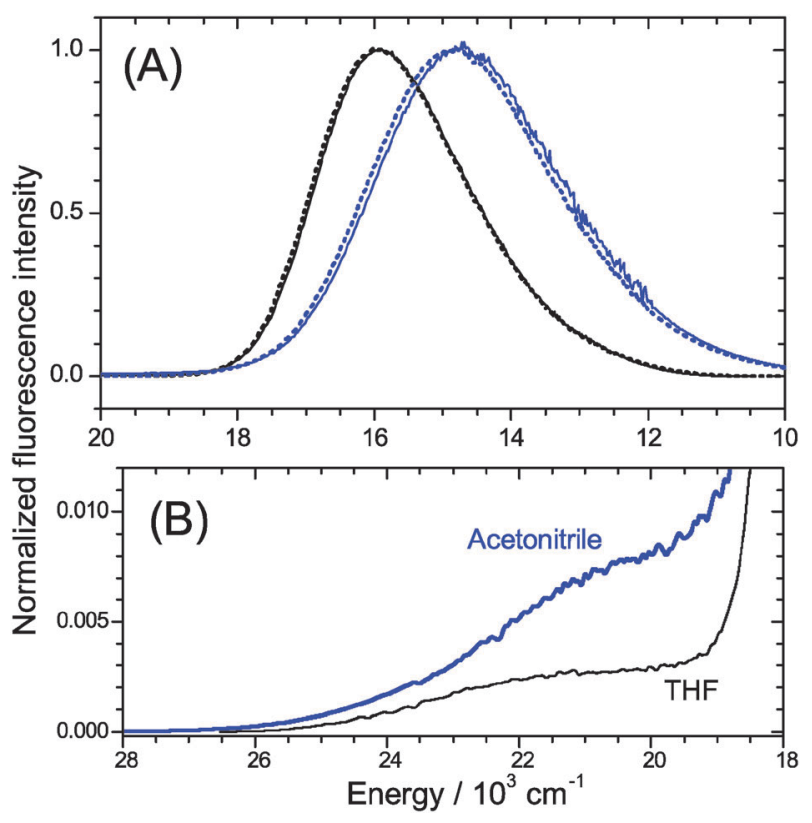

Fig. 3 (A) Steady-state fluorescence spectra of D149 after $S_{0} \rightarrow S_{1}$ (dotted lines) and $\mathrm{S}_{0} \rightarrow \mathrm{S}_{2}$ (solid lines) excitation for THF (black) and acetonitrile (blue). (B) Magnification of the fluorescence spectra after $\mathrm{S}_{0} \rightarrow \mathrm{S}_{2}$ excitation shown in (A) in the region where $\mathrm{S}_{2} \rightarrow \mathrm{S}_{0}$ emission is expected. Spectra in (A) below $11800 \mathrm{~cm}^{-1}$ have been extrapolated.

estimated errors from the fitting procedures. A global kinetic analysis will be presented in Section 3.3.

We commence with the PSCP experiments for acetonitrile (Fig. 4): the upper plot shows the early-time dynamics between -0.1 and $0.22 \mathrm{ps}$ in steps of $40 \mathrm{fs}$. Here, one observes the negative ground state bleach (GSB, $\mathrm{S}_{0} \rightarrow \mathrm{S}_{n}$ ) of D149, with a characteristic double peak structure resembling the inverted steady-state absorption spectrum (shown as a dashed blue line in the bottom panel). In addition, nascent stimulated emission (SE) is visible to the red of the GSB, arising from the $\mathrm{S}_{1}$ state prepared by photoexcitation. It is superimposed by weaker structured Raman contributions, which disappear as soon as the pump and probe pulses no longer overlap. Furthermore, there is excited state absorption (ESA) over the whole spectral window. In the GSB region, the superimposed ESA contribution is immediately noticeable from the positive signal at around $430 \mathrm{~nm}$ (probably due to a transition to a higher-lying electronically excited state: $\mathrm{S}_{1} \rightarrow \mathrm{S}_{n}$ ). The ESA is much more pronounced to the red $(570-770 \mathrm{~nm})$. In this spectral region, one readily observes the beginning of a spectral development, which is due to solvation dynamics caused by the ultrafast reorientation of acetonitrile molecules, which respond to the altered charge distribution when D149 is excited to $S_{1}$. This response is reported by the transient red-shift of the SE band of D149.

The solvation dynamics becomes more obvious in the middle panel of Fig. 4, which summarizes the spectral evolution from $0.4 \mathrm{ps}$ to $1.2 \mathrm{ps}$ in $200 \mathrm{fs}$ steps, and from $1.5 \mathrm{ps}$ to $3.0 \mathrm{ps}$ in $500 \mathrm{fs}$ steps. The SE moves further to the red to approach its equilibrium position (compare the steady-state SE spectrum in the bottom panel), and this process results in a negative signal between 650 and $770 \mathrm{~nm}$. At the same time, the superimposed strong $\mathrm{S}_{1} \rightarrow \mathrm{S}_{n}$ ESA peak centered at $c a .600 \mathrm{~nm}$

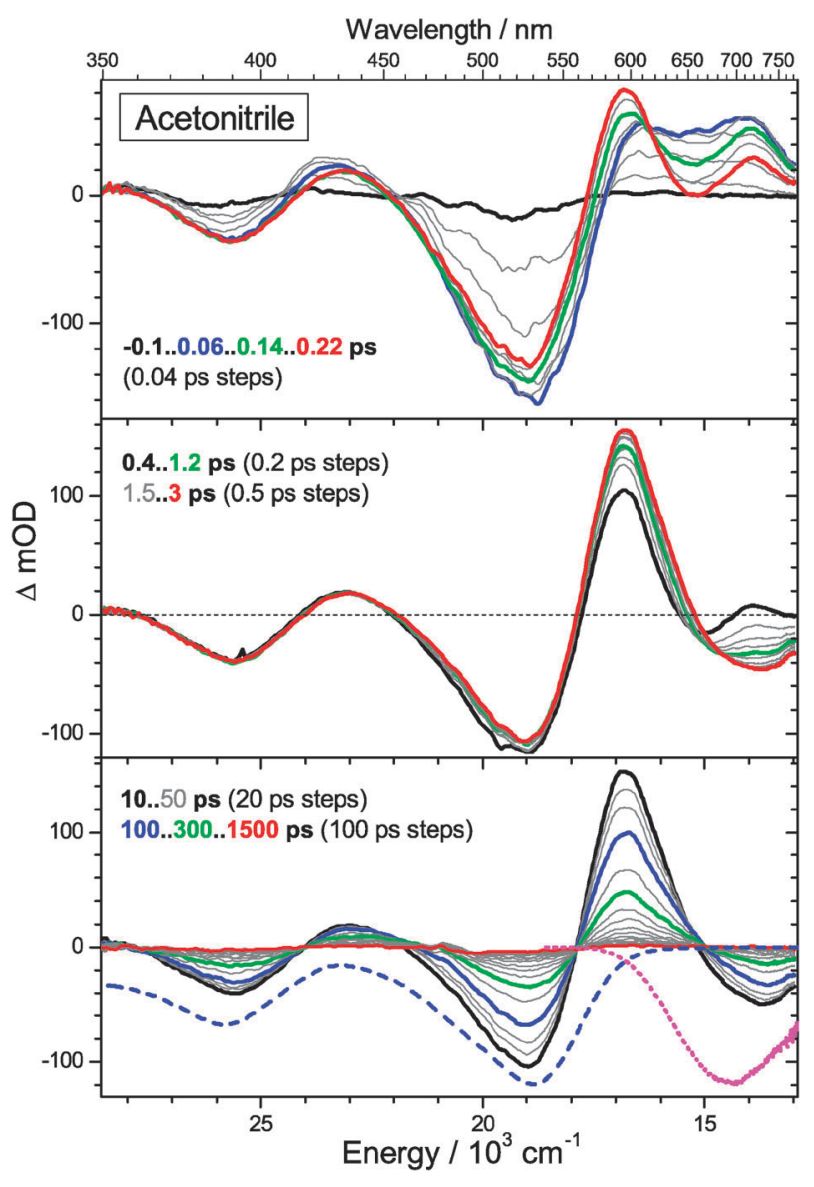

Fig. 4 Transient PSCP absorption spectra of D149 in acetonitrile. Excitation: $476 \mathrm{~nm}$ (cross correlation $100 \mathrm{fs}$ ): (upper panel) $-0.10-0.22 \mathrm{ps}$ with 40 fs steps; (middle panel) $0.4-1.2$ ps with 0.2 ps steps and $1.5-3$ ps with 0.5 ps steps; (lower panel) $10-50$ ps with 20 ps steps and $100-1500$ ps with 100 ps steps. Some transient spectra are plotted as thick colored lines for guidance. For comparison, the inverted and scaled steady-state absorption and the scaled steady-state stimulated emission spectrum is shown in the lower panel as blue dashed and magenta dotted lines, respectively.

band rises further up, due to the disappearing SE in that spectral region. The solvation dynamics in acetonitrile have completely ceased by $3 \mathrm{ps}$, which is the expected timescale in this solvent. ${ }^{28}$

The bottom panel shows the final decay of the $\mathrm{S}_{1} \rightarrow \mathrm{S}_{n}$ ESA bands and the concomitant filling-up of the GSB with a time constant of $\tau_{1}=280 \mathrm{ps}$, which must be dominated by $\mathrm{S}_{1} \rightarrow \mathrm{S}_{0}$ internal conversion (IC) with a minor contribution of radiative decay. We note that the study of Fakis et al. reported a $\mathrm{S}_{1}$ lifetime of $220 \mathrm{ps}$ in acetonitrile. ${ }^{14}$ This is considerably shorter than in our experiments, and is due to the fact that the fluorescence up-conversion transients in that study were measured up to $400 \mathrm{ps}$, which is too short to determine an accurate $S_{1}$ lifetime. In addition to the $S_{1}$ spectral decay, we see a barely noticeable change in the ESA band structure, with a time constant of $19 \mathrm{ps}$. The timescale is consistent with the removal of vibrational excess energy from the $S_{1}$ state by collisions with solvent molecules, ${ }^{20,29,30}$ but $\mathrm{S}_{1}$ structural relaxation could be also responsible for this change.

Inspecting the dynamics of D149 in the other two solvents supports the picture outlined above. For methanol (Fig. 5), 


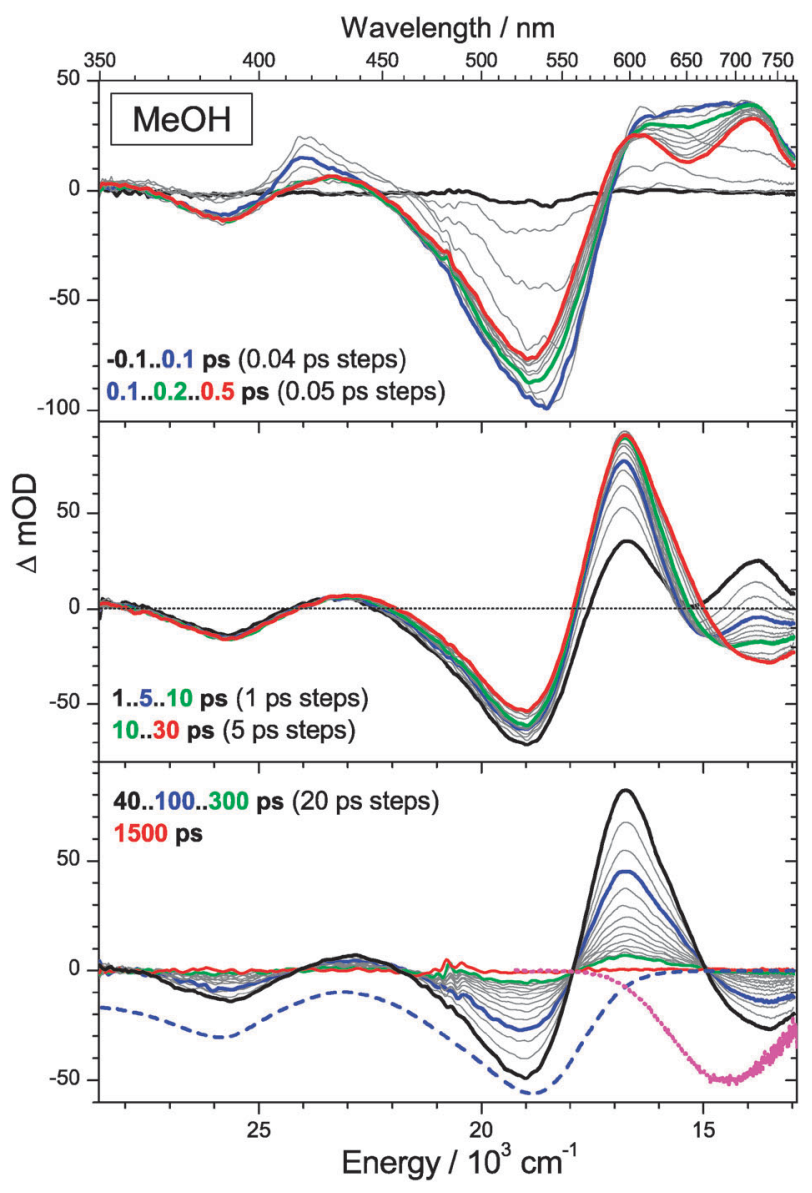

Fig. 5 Transient PSCP absorption spectra of D149 in methanol. Excitation: $479 \mathrm{~nm}$ (cross correlation $90 \mathrm{fs}$ ): (upper panel) $-0.1-0.1 \mathrm{ps}$ with 40 fs steps and $0.1-0.5$ ps with 50 fs steps; (middle panel) $1-10 \mathrm{ps}$ with 1 ps steps and $10-30$ ps with 5 ps steps; (lower panel) 40-300 ps with 20 ps steps and 1500 ps. Some transient spectra are plotted as thick colored lines for guidance. For comparison, the inverted and scaled steady-state absorption and the scaled steady-state stimulated emission spectrum is shown in the lower panel as blue dashed and magenta dotted lines, respectively. Increased noise around $479 \mathrm{~nm}$ (see e.g. lower panel) is due to an imperfect stray light subtraction.

the qualitative development is very similar, yet both solvation and $\mathrm{S}_{1} \rightarrow \mathrm{S}_{0}$ decay proceed on different timescales compared to acetonitrile. Multiexponential solvation dynamics containing fast and slow time constants are found (see the top and middle panels and the global analysis below). Once solvation dynamics has ceased (see the bottom panel which shows clear isosbestic points), the resulting spectrum exhibits SE and ESA band shapes similar to acetonitrile. This is consistent with the comparable Stokes shift of D149 in both solvents, resulting in similar steady-state SE spectra. Interestingly, the lifetime of the $S_{1}$ state in methanol is much shorter $\left(\tau_{1}=99 \mathrm{ps}\right)$, pointing toward an acceleration of the IC process, as will be discussed below. Concerning the additional 19 ps ESA dynamics in $\mathrm{S}_{1}$ found in acetonitrile, it might be also present in methanol. However, it is difficult to separate it from the slowest component of the methanol solvation dynamics which is $11.9 \mathrm{ps}$.

The data in THF provide an example for D149 relaxation in a mid-polar solvent with fast solvation dynamics (Fig. 6). Again, an ultrafast transient red-shift of the SE is observed

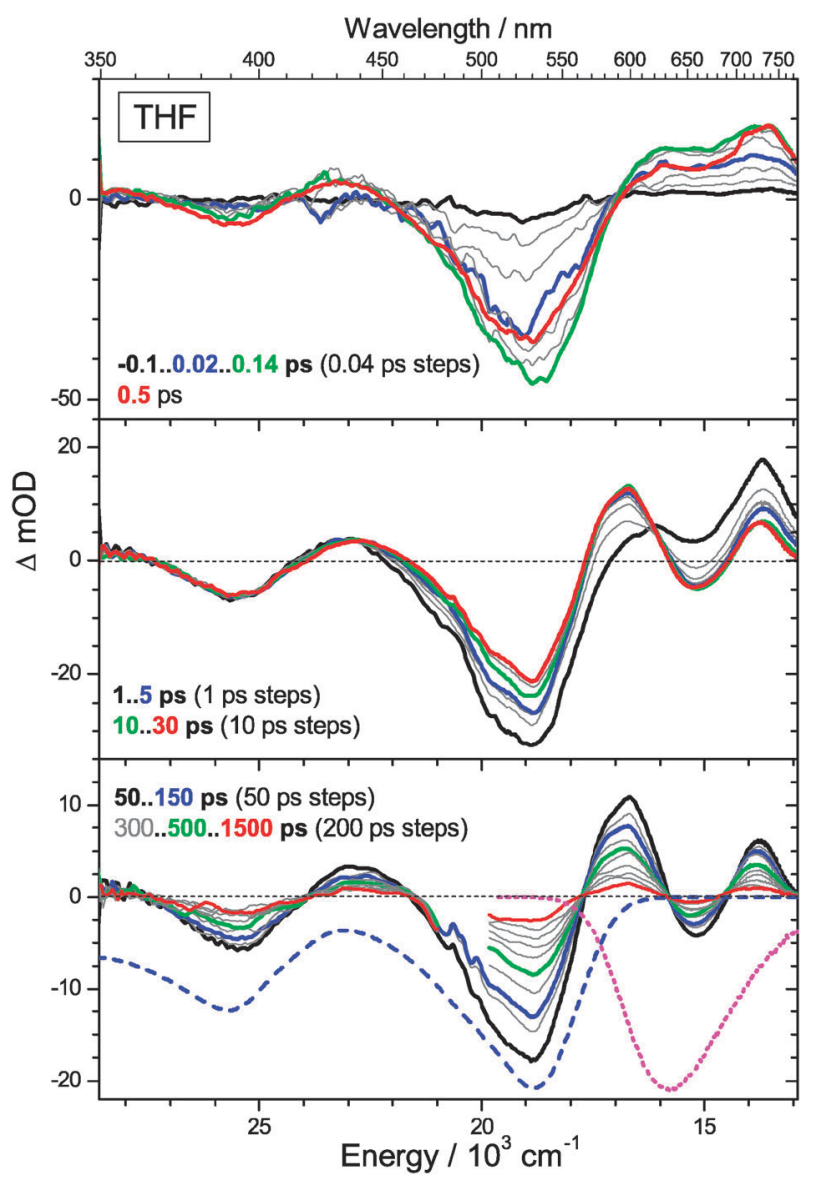

Fig. 6 Transient PSCP absorption spectra of D149 in THF. Excitation: $484 \mathrm{~nm}$ (cross correlation $100 \mathrm{fs}$ ): (upper panel) $-0.10-0.14 \mathrm{ps}$ with $40 \mathrm{fs}$ steps and $0.5 \mathrm{ps}$; (middle panel) $1-5 \mathrm{ps}$ with $1 \mathrm{ps}$ steps and $10-30 \mathrm{ps}$ with 10 ps steps; (lower panel) $50-150$ ps with 50 ps steps and $300-1500$ ps with 200 ps steps. Some transient spectra are plotted as thick colored lines for guidance. For comparison, the inverted and scaled steady-state absorption and the scaled steady-state stimulated emission spectrum is shown in the lower panel as blue dashed and magenta dotted lines, respectively. Note that in the bottom panel the wavelength range 480-510 $\mathrm{nm}$ has been omitted for a few spectra for the sake of clarity, because there is excessive noise due to an imperfect stray light correction.

in the top and middle panels (which is completed in less than $10 \mathrm{ps}$ ), but the spectral appearance is different from that in acetonitrile and methanol, because the Stokes shift in THF is much smaller. The negative SE signal is therefore located more to the blue (negative peak at $660 \mathrm{~nm}$ ). As a result, the ESA peak at $596 \mathrm{~nm}$ is much smaller than in polar solvents, and another ESA peak is visible further to the red, centered at $726 \mathrm{~nm}$, which is otherwise overwhelmed by the more red-shifted SE in polar solvents. The lifetime of the $\mathrm{S}_{1}$ state is much longer than in acetonitrile and methanol and was independently determined from our TCSPC measurements. A representative trace is shown in Fig. 7, which can be nicely modeled by a clean monoexponential decay with $\tau_{1}=720$ ps. Corresponding TCSPC experiments for D149 in acetonitrile yielded a trace which is slightly wider than the IRF of the TCSPC setup and can be well represented by the decay constant $\tau_{1}=280 \mathrm{ps}$ from the PSCP experiments. In this case, the time-resolution 
Table 2 Summary of global analysis results for D149 in organic solvents

\begin{tabular}{|c|c|c|c|c|c|c|c|c|}
\hline Solvent & $\lambda_{\text {pump }}{ }^{a} / \mathrm{nm}$ & $\tau_{\mathrm{CC}}^{b} / \mathrm{fs}$ & $\tau_{2}^{c} / \mathrm{ps}$ & $\tau_{1}{ }^{c} / \mathrm{ps}$ & $\tau_{\text {relax }}{ }^{d} / \mathrm{ps}$ & $\tau_{\mathrm{solv}, 1}{ }^{e} / \mathrm{ps}$ & $\tau_{\mathrm{solv}, 2}{ }^{e} / \mathrm{ps}$ & $\tau_{\text {solv }, 3}{ }^{e} / \mathrm{ps}$ \\
\hline Acetonitrile & $\begin{array}{l}476 \\
377.5\end{array}$ & $\begin{array}{l}100 \\
130\end{array}$ & $\overline{0.4} \pm 0.1$ & $280 \pm 10$ & $\underset{f}{19} \pm 10$ & 0.089 & 0.63 & - \\
\hline Methanol & $\begin{array}{l}479 \\
377.5\end{array}$ & $\begin{array}{r}90 \\
130\end{array}$ & $\overline{0.3} \pm 0.1$ & $99 \pm 5$ & $f_{f}^{f}$ & 0.12 & 0.99 & 11.9 \\
\hline $\begin{array}{l}\text { Ethanol } \\
\text { Acetone }\end{array}$ & $\begin{array}{l}377.5 \\
377.5 \\
484\end{array}$ & $\begin{array}{l}130 \\
130 \\
100\end{array}$ & - & $\begin{array}{l}178 \pm 10 \\
540 \pm 20\end{array}$ & $\begin{array}{l}f \\
{ }^{f} f \\
30+10\end{array}$ & $\begin{array}{l}0.39 \\
0.187 \\
0.228\end{array}$ & $\begin{array}{l}5.03 \\
1.09 \\
152\end{array}$ & $\begin{array}{l}29.6 \\
- \\
\end{array}$ \\
\hline THF & $\begin{array}{l}484 \\
377.5\end{array}$ & $\begin{array}{l}100 \\
130\end{array}$ & $\overline{0.6} \pm 0.3$ & $720 \pm 20$ & $\underset{f}{30} \pm 10$ & 0.228 & 1.52 & - \\
\hline
\end{tabular}

${ }^{a}$ PSCP experiment: $S_{0} \rightarrow S_{1}$ excitation at 476-484 nm, UV-pump VIS-probe experiment: $S_{0} \rightarrow S_{2}$ excitation at $377.5 \mathrm{~nm} .{ }^{b}$ Pump-probe crosscorrelation of the experiment extracted from the width of the "coherent artifact". ${ }^{c} S_{2}$ and $S_{1}$ lifetimes $\tau_{2}$ and $\tau_{1}$ from transient absorption experiments. In the case of THF the $\mathrm{S}_{1}$ lifetime of 720 ps was extracted from TCSPC experiments. The corresponding TCSPC value for chloroform is $(800 \pm 20)$ ps. ${ }^{d}$ Time constant for vibrational relaxation in the $\mathrm{S}_{1}$ state due to collisions with the solvent (or possibly structural relaxation). ${ }^{e}$ Time constants for solvent relaxation taken from ref. 28 (acetonitrile, ethanol, acetone and THF) and ref. 31 (methanol). Note: $\tau_{\text {solv, } 1}$ for methanol is for a Gaussian function, whereas in all other cases exponential functions are used. ${ }^{f}$ Time constant cannot be determined, either due to timescale overlap with $\tau_{\text {solv }, 3}$ (PSCP, methanol) or due to insensitivity of the transients at $755 \mathrm{~nm}$ to this process (experiments with single probe wavelength).

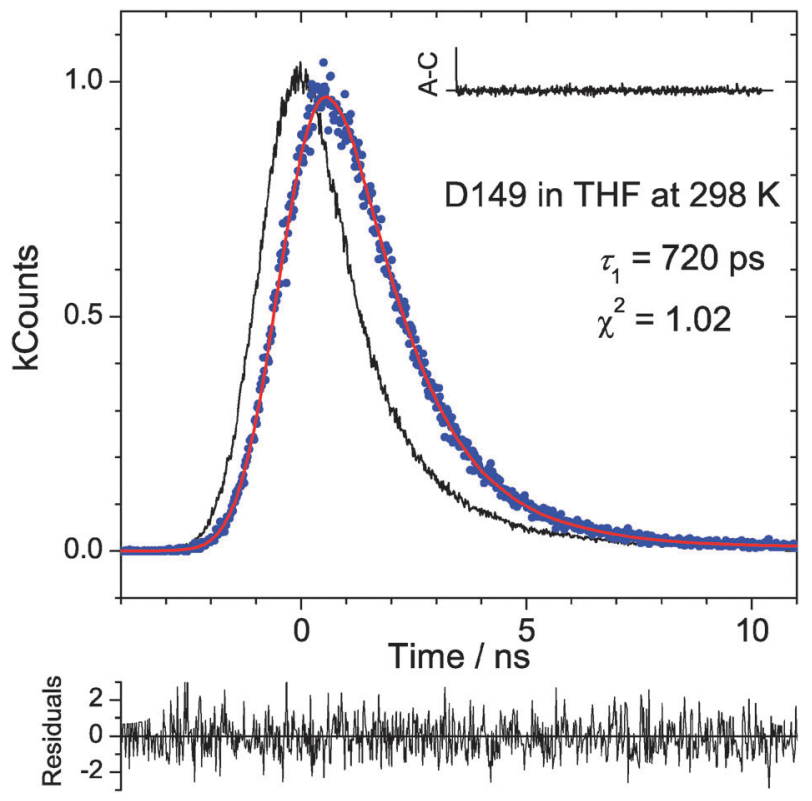

Fig. 7 Time-correlated single photon counting results (blue circles) for D149 in THF at $298 \mathrm{~K}$. Excitation: $337 \mathrm{~nm}$, detection: $610 \mathrm{~nm}\left(\mathrm{~S}_{1} \rightarrow \mathrm{S}_{0}\right)$. The red line is the best fit with $\tau_{1}=720 \mathrm{ps}\left(\chi^{2}=1.02\right)$ employing the instrument response function obtained from the scattering solution (black line). The inset shows the autocorrelation (A-C) trace and the lower plot the weighted residuals of the fit.

of the TCSPC setup does not permit an accurate independent determination of $\tau_{1}$.

\subsection{Global kinetic analysis of PSCP data}

The transient PSCP spectra in Fig. 4-6 were subjected to a global kinetic analysis based on the simple $S_{1} \rightarrow S_{0}$ model. Solvation dynamics and collisional deactivation of the $S_{1}$ state were conveniently modeled by a time-dependent $S_{1}$ spectrum, as described in our earlier studies of other molecules. ${ }^{19,20,30}$ In contrast to the carotene systems previously investigated by us, ${ }^{20,30}$ the influence of vibrationally hot molecules in $S_{0}$ on the transient spectra can be safely neglected, because the lifetime of the $\mathrm{S}_{1}$ state is fairly long compared to collisional relaxation: $\tau_{1}$ is in the range 99 to $800 \mathrm{ps}$ depending on the solvent, whereas $\tau_{\text {relax }}$ is $c a$. 19-30 ps (estimate based on the values for $\mathrm{S}_{1}$ ). In the case of THF, the decay $\mathrm{S}_{1} \rightarrow \mathrm{S}_{0}$ is fairly slow, and therefore it cannot be covered completely within the timewindow of the PSCP experiments. Therefore the $\mathrm{S}_{1}$ lifetime was determined from our TCSPC experiments and kept constant in the course of the global analysis.

The resulting time-dependent $\mathrm{S}_{1}$ spectra for D149 in acetonitrile, methanol and THF are shown in Fig. 8. In all cases, the time-dependence is dominated by the transient Stokes shift of the SE. In contrast, the concomitant shift of the ESA peak at around $600 \mathrm{~nm}$ is minor suggesting that the energy gap between the $S_{1}$ and $S_{n}$ states involved in the transition is not changing appreciably in the course of the solvent relaxation. In all cases, the solvation dynamics can be nicely and consistently modeled with the time constants of Horng et al. (acetonitrile, THF) ${ }^{28}$ and Ernsting and co-workers (methanol), ${ }^{31}$ for coumarin 153 and rhodamine 110, respectively. The values are summarized in Table 2: a fast biexponential relaxation in acetonitrile ( 89 and $630 \mathrm{fs}$ ) and THF (228 fs and $1.52 \mathrm{ps)}$ ) and a more complex response in methanol with one Gaussian and two exponential functions (120 fs, $990 \mathrm{fs}$ and $11.9 \mathrm{ps}$, respectively). As mentioned above, the additional weak spectral development after completion of the solvent dynamics can be tentatively ascribed to collisional cooling (or possibly also structural relaxation) of the $\mathrm{S}_{1}$ state, which takes $19 \mathrm{ps}$ in acetonitrile and $30 \mathrm{ps}$ in THF. It might be also present in methanol, however a reliable time constant cannot be extracted due to the overlap with the slowest part of the solvation dynamics.

In Fig. 9, examples for kinetic traces of D149 in methanol are shown for selected wavelengths together with fit results (solid black lines) including the contributions from $\mathrm{S}_{1}$ (green lines) and $\mathrm{S}_{0}$ (red lines). The traces at 390 and $500 \mathrm{~nm}$ show a steep decay (GSB) and a recovery with a time constant of $99 \mathrm{ps}$ $\left(\mathrm{S}_{1} \rightarrow \mathrm{S}_{0}\right)$, with additional weak curvature in the early part of the transients, which is due to solvation dynamics. At $600 \mathrm{~nm}$, $\mathrm{S}_{1} \rightarrow \mathrm{S}_{n}$ ESA is formed, whereas at $720 \mathrm{~nm}$ first $\mathrm{S}_{1} \rightarrow \mathrm{S}_{n}$ ESA and then $\mathrm{S}_{1} \rightarrow \mathrm{S}_{0} \mathrm{SE}$ develop. In both cases, the final decay is 


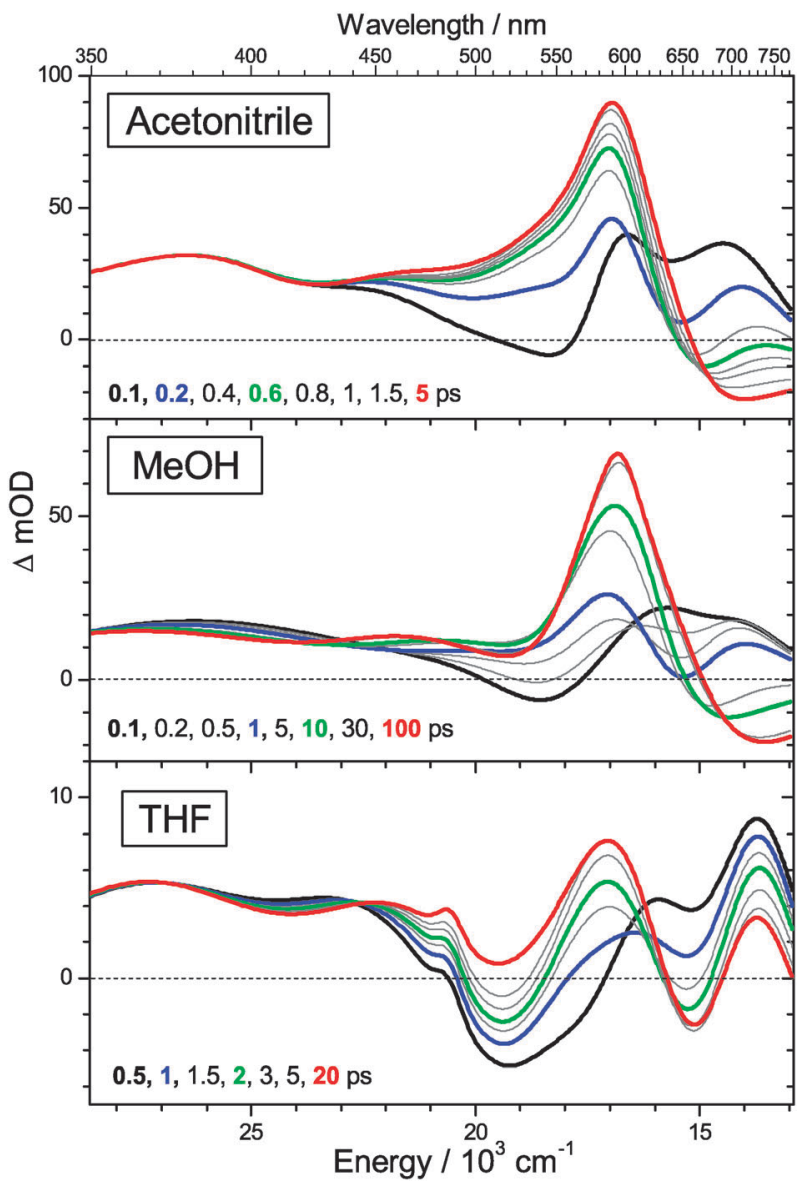

Fig. $8 \mathrm{~S}_{1}$ spectra of D149 in (A) acetonitrile, (B) methanol and (C) THF as obtained from the global kinetic analysis. The corresponding time for each spectrum is given in each panel.

also due to the recovery of $S_{0}$ with a time constant of 99 ps. The superimposed $S_{1}$ solvation dynamics become particularly clear in the insets of the 600 and $720 \mathrm{~nm}$ traces, where the magnification shows the characteristic curvature, which arises from the time-dependent Stokes shift of the SE band.

Additional transient absorption experiments have been performed by employing excitation at $377.5 \mathrm{~nm}\left(\mathrm{~S}_{0} \rightarrow \mathrm{S}_{2}\right)$ and probing in the SE band at $755 \mathrm{~nm}$. These experiments will be discussed in more detail in Section 3.4, but at this point we already mention that the measurements in two additional solvents (acetone and ethanol) can also be nicely described by solvation times from the literature. ${ }^{28}$ These values are reported in Table 2 together with the $\mathrm{S}_{1}$ lifetime, which is $178 \mathrm{ps}$ in ethanol and $540 \mathrm{ps}$ in acetone, respectively. Therefore, the lifetime $\tau_{1}$ of the $S_{1}$ state approximately correlates with polarity, e.g. acetonitrile $\left(\tau_{1}=280 \mathrm{ps}\right)<$ acetone $\left(\tau_{1}=\right.$ $540 \mathrm{ps})<\operatorname{THF}\left(\tau_{1}=720 \mathrm{ps}\right)<$ chloroform $\left(\tau_{1}=800 \mathrm{ps}\right)$. However, interestingly, in protic solvents the lifetime is much shorter [methanol $\left(\tau_{1}=99 \mathrm{ps}\right)<\operatorname{ethanol}\left(\tau_{1}=178 \mathrm{ps}\right)<$ acetonitrile $\left(\tau_{1}=280 \mathrm{ps}\right)$ ], suggesting a substantial influence of hydrogen bonding on the dynamics. A similar acceleration of nonradiative processes in alcohols was observed for other molecules, such as 4 -aminophthalimide. ${ }^{32}$ It is accompanied by an increased Stokes shift in protic solvents, ${ }^{33}$ which is also observed for D149 (Table 1).

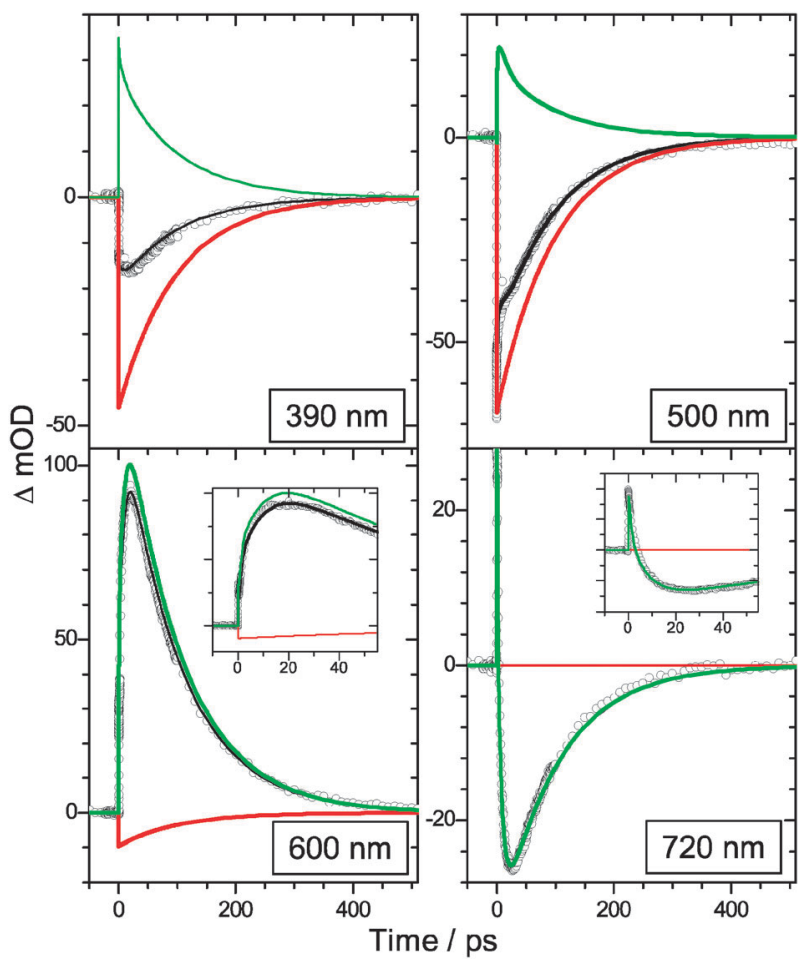

Fig. 9 Kinetic traces for D149 in methanol at four representative probe wavelengths $(390,500,600$ and $720 \mathrm{~nm}$ ): (circles) experimental PSCP data from Fig. 5; (black line) simulation results from global kinetic analysis with individual contributions from $\mathrm{S}_{1}$ (green line) and $\mathrm{S}_{0}$ (red line). In the lower two plots, the insets show magnifications of the transients at earlier times to highlight the curvature in the kinetic traces which is due to solvation dynamics.

\subsection{Lifetime of the $S_{2}$ state}

In addition to the PSCP experiments employing $\mathrm{S}_{0} \rightarrow \mathrm{S}_{1}$ excitation we have carried out transient absorption measurements with $\mathrm{S}_{0} \rightarrow \mathrm{S}_{2}$ excitation at $377.5 \mathrm{~nm}$ and single wavelength probing at $755 \mathrm{~nm}$, to determine the lifetime of the $\mathrm{S}_{2}$ state. An example for the short-time dynamics in methanol is presented in Fig. 10, which contains the transient absorption signals for $\mathrm{S}_{1}$ excitation (red circles, PSCP) and $\mathrm{S}_{2}$ excitation (black circles) normalized to the same amplitude of the SE (for both transients occurring at $c a .25$ ps, not shown here for the sake of clarity). $S_{2}$ excitation obviously results in additional $\mathrm{S}_{2} \rightarrow \mathrm{S}_{n} \mathrm{ESA}$, which is responsible for the increased amplitude at early times. The $S_{2} \rightarrow S_{n}$ ESA decays by $S_{2} \rightarrow S_{1}$ IC to form $\mathrm{S}_{1} \rightarrow \mathrm{S}_{n}$ ESA, which later on becomes dominated by $\mathrm{S}_{1} \rightarrow \mathrm{S}_{0} \mathrm{SE}$, once solvation dynamics has sufficiently progressed (compare e.g. the $720 \mathrm{~nm}$ transient in Fig. 9).

It is obvious that the $S_{2}$ lifetime has a critical impact on the appearance of the transient, because it influences the width of the peak, its early-time decay, and (to a much smaller extent) also the signal rise. Using the best fit parameters from the PSCP experiments for methanol in Table 2, the $\mathrm{S}_{2}$ lifetime and absorption coefficient were simultaneously varied to obtain an optimized fit, which was reached for $\tau_{2}=0.3 \mathrm{ps}$ (black solid line in Fig. 10). Contributions of $S_{2}$ and $S_{1}$ to the fit are shown as solid blue and solid green lines, respectively. Shorter (longer) lifetimes result in too narrow (broad) simulations, as shown in the figure. Using a similar approach we obtain a lifetime of 


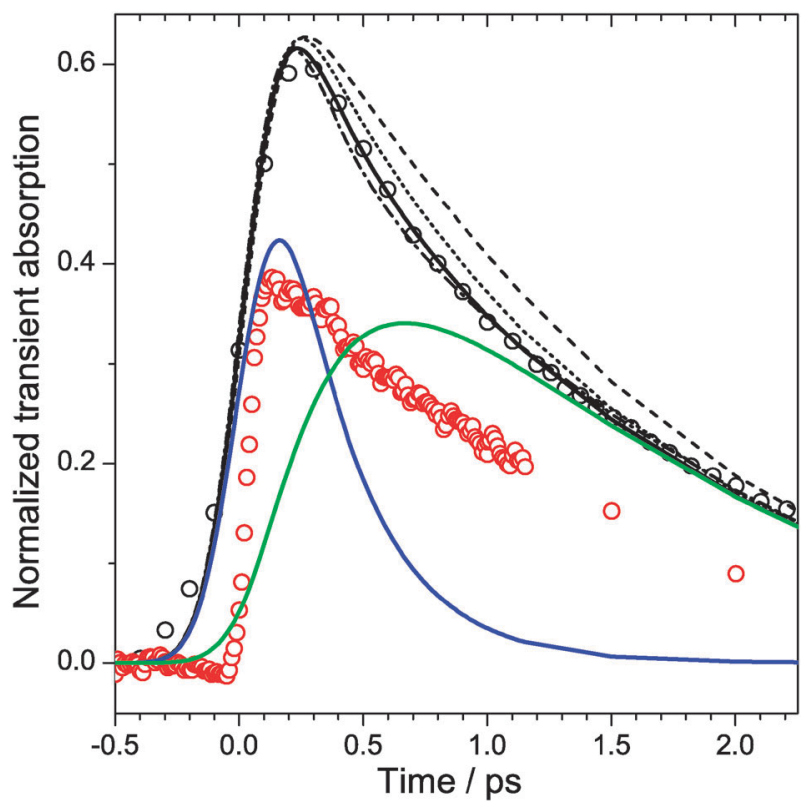

Fig. 10 Comparison of transient absorption profiles of D149 in methanol at $755 \mathrm{~nm}$ obtained after $\mathrm{S}_{1}$ excitation at $479 \mathrm{~nm}$ (PSCP, red circles) and $\mathrm{S}_{2}$ excitation at $377.5 \mathrm{~nm}$ (black circles). The black

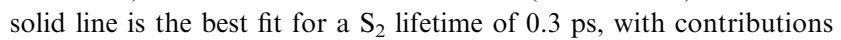
from $S_{2}$ (blue solid line) and $S_{1}$ (green solid line). Other black fit lines are from a sensitivity analysis using other $\mathrm{S}_{2}$ lifetimes: 0.2 ps (dashdotted line), 0.4 ps (dotted line) and 0.6 ps (dashed line).

$0.4 \mathrm{ps}$ in acetonitrile and (less accurate due to a worse signalto-noise ratio) $c a$. $0.6 \mathrm{ps}$ in THF. Our values are in good agreement with results of Fakis et al., who reported 0.44 ps (toluene) and 0.31 ps (acetonitrile) based on their up-conversion measurements of the $\mathrm{S}_{2}$ fluorescence. ${ }^{14}$

Our values can be compared with results from another much more approximate indirect approach for obtaining the $S_{2}$ lifetime by using the area ratio of the $S_{2}$ and $S_{1}$ fluorescence bands, which can be estimated from deconvolution of the emission bands in Fig. 3. We note that this deconvolution is not straightforward because the two emission bands overlap. Assuming a simple kinetic scheme involving the nonradiative IC steps $\mathrm{S}_{2} \rightarrow \mathrm{S}_{1}$ and $\mathrm{S}_{1} \rightarrow \mathrm{S}_{0}$ as well as the radiative decays from $S_{2}$ and $S_{1}$ one arrives at:

$$
\frac{A_{\mathrm{F}}\left(\mathrm{S}_{2} \rightarrow \mathrm{S}_{0}\right)}{A_{\mathrm{F}}\left(\mathrm{S}_{1} \rightarrow \mathrm{S}_{0}\right)}=\frac{\Phi_{\mathrm{F}}\left(\mathrm{S}_{2} \rightarrow \mathrm{S}_{0}\right)}{\left(1-\Phi_{\mathrm{F}}\left(\mathrm{S}_{2} \rightarrow \mathrm{S}_{0}\right)\right) \Phi_{\mathrm{F}}\left(\mathrm{S}_{1} \rightarrow \mathrm{S}_{0}\right)}
$$

In eqn (1) the $A_{\mathrm{F}}$ denotes the areas of the corresponding fluorescence bands. We obtain the area ratio from the deconvolution of the emission bands in Fig. 3(B) as $9.8 \times 10^{-3}$ in acetonitrile and $6.8 \times 10^{-3}$ in THF. The fluorescence quantum yield $\Phi_{\mathrm{F}}$ for the state $\mathrm{S}_{i}$ is given as

$$
\Phi_{\mathrm{F}}\left(\mathrm{S}_{i} \rightarrow \mathrm{S}_{0}\right)=\frac{\tau_{i}}{\tau_{\mathrm{rad}, i}}
$$

Here, $\tau_{\mathrm{rad}, i}$ and $\tau_{i}$ are the radiative and fluorescence lifetimes for the state $i$. We have roughly estimated the radiative lifetime of the $S_{2}$ and $S_{1}$ states using the Strickler-Berg formula for the two examples given in Fig. $3 .^{34}$ Employing our absorption and fluorescence spectra, an absorption coefficient of
$68700 \mathrm{~L} \mathrm{~mol}^{-1} \mathrm{~cm}^{-1}$ for the maximum of the $\mathrm{S}_{0} \rightarrow \mathrm{S}_{1}$ band, ${ }^{2}$ and wavelength-dependent refractive indices from the literature, ${ }^{35}$ we obtain for the $S_{1}$ state $6.2 \mathrm{~ns}$ in acetonitrile and $4.3 \mathrm{~ns}$ in THF. The corresponding values for the $\mathrm{S}_{2}$ state are $4.3 \mathrm{~ns}$ in acetonitrile and $2.7 \mathrm{~ns}$ in THF. With D149 in THF, the direct calculation of $\tau_{\mathrm{rad}, i}$ from eqn (2) employing the measured $\Phi_{\mathrm{F}}(0.140$ in nitrogen-saturated $\mathrm{THF}$ at $298 \mathrm{~K})$ and $\tau_{1}=720 \mathrm{ps}$ (Table 2) provides a value of $5.2 \mathrm{~ns}$. This confirms that the Strickler-Berg approach provides a reasonable estimate of $\tau_{\text {rad }}$ for D149. Using these results and our measured $S_{1}$ lifetimes $\tau_{1}=280$ and 720 ps in acetonitrile and THF, respectively, we arrive at $\tau_{2}=1.5 \mathrm{ps}$ in acetonitrile and $3.1 \mathrm{ps}$ in THF. While these time constants suggest a much faster decay of $S_{2}$ compared to $S_{1}$, they are still substantially larger than our direct determination of $\tau_{2}$ from transient absorption spectroscopy. This result means that the observed $\mathrm{S}_{2}$ emission consists in fact mainly of the emission from a small amount of an impurity. This interpretation is also supported by our TCSPC experiments with lamp excitation at $337 \mathrm{~nm}$, where the blue emission band of D149 in THF shows a double exponential decay at $460 \mathrm{~nm}$ : the first component has a very small time constant (far below TCSPC time resolution), and the second component is very weak (relative amplitude: $1.9 \times 10^{-4}$ ) and long-lived (decay time constant ca. $2.0 \mathrm{~ns}$ ). The fast and slow components contribute about 30 and $70 \%$ to the overall emission intensity at $460 \mathrm{~nm}$, respectively. The TCSPC experiment is practically not affected by photodecomposition due to the very low excitation power (i.e. photoproducts are not reexcited). This fact allows us to assign the fast component to $\mathrm{S}_{2}$ emission of D149 and the long component to the fluorescence of an impurity.

We also note that our experiments employing $\mathrm{S}_{2}$ excitation suggest the presence of an ultrafast photoinduced process in D149, which must proceed either directly from $\mathrm{S}_{2}$ or at high excess energies in $\mathrm{S}_{1}$ : for instance, the transient absorption profiles at $755 \mathrm{~nm}$ exhibit a small (few percent) residual absorption, which is not present after $\mathrm{S}_{1}$ excitation (not shown here). In the same experiments, prolonged laser excitation in the $S_{2}$ band at $377.5 \mathrm{~nm}$ leads to a change in the steady-state absorption spectrum of the solution, where the $S_{0} \rightarrow S_{1}$ band is asymmetrically broadened (reaches further to the red) by a few nm (the exact value depending on the solvent) and, at the same time, the $\mathrm{S}_{2}$ band reduces in intensity by about $10 \%$. Our reported $\mathrm{S}_{2}$ lifetime therefore possibly contains contributions from $S_{2} \rightarrow S_{1}$ IC and another nonradiative pathway. Interestingly however, the lifetime of the $S_{1}$ state is independent of excitation wavelength. Indications for the formation of a photoproduct are also obtained in experiments with sub-400 nm excitation in the $\mathrm{S}_{0} \rightarrow \mathrm{S}_{2}$ band of D149 in solution by cw lamp illumination with appropriate filters. In this case, the absorption spectra reach a photostationary state within a few minutes. An example is given in the ESI. $\dagger$ Recall also that the $\mathrm{S}_{1} \rightarrow \mathrm{S}_{0}$ fluorescence band shows a small red-shift when switching from $S_{1}$ to $S_{2}$ excitation (Fig. 3(A)). All these observations could be, e.g., tentatively explained by a photoisomerization reaction of D149 after $S_{2}$ excitation, yet a full explanation of this complex behavior certainly requires further experiments, which are beyond the scope of the current paper and are currently ongoing in our laboratories. 


\section{Conclusions}

We have presented a comprehensive study of the photoinduced dynamics of the indoline dye D149 in a range of organic solvents. The dynamics after $S_{1}$ photoexcitation are fairly simple for such a complex molecule. The increase of the D149 dipole moment upon photoexcitation triggers solvation dynamics on characteristic timescales, which manifest themselves in a transient Stokes shift in the red to near IR region and an accompanying increase of a superimposed prominent ESA band at around $600 \mathrm{~nm}$. The decay of the $\mathrm{S}_{1}$ state proceeds on a timescale between 99 ps (methanol) and 800 ps (chloroform), and is dominated by internal conversion. In protic solvents, the relaxation is considerably faster than in aprotic solvents of similar polarity, suggesting the influence of hydrogen-bonding, which is likely also responsible for the trends in the Stokes shifts. Transient absorption experiments after $\mathrm{S}_{2}$ photoexcitation reveal an ultrafast decay of this state on a sub-picosecond timescale and also the formation of a photoproduct, which will require further characterization.

Regarding the already demonstrated successful application of this dye in photoelectrochemical solar cells the following comments can be made: the $S_{1}$ lifetime of the "isolated" dye is long enough such that electron injection into semiconductor oxide electrodes (which likely occurs on a $100 \mathrm{fs}$ timescale, possibly with additional picosecond components) ${ }^{14}$ should efficiently compete with the intramolecular relaxation of the $\mathrm{S}_{1}$ state of D149, resulting in a high quantum yield for electron injection. The observed generation of a photoproduct after $\mathrm{S}_{2}$ excitation is probably not desirable: it might convert the sensitizer into an inactive form, especially under long-term illumination, but in the most favourable case the photoproduct (which has a similar spectrum) might inject electrons as well. Such an unwanted side-reaction could however be efficiently suppressed, once the dye is attached to a semiconductor oxide electrode, as has been demonstrated in the case of merocyanine dyes. $^{36}$ Investigations along these lines are currently underway in our laboratories.

\section{Acknowledgements}

We would like to thank N.P. Ernsting and J.L. Pérez Lustres for extensive help during the implementation of the PSCP setup, J. Troe and A.M. Wodtke for on-going generous support, as well as R. Bürsing for excellent technical assistance during the experiments. We are also indebted to K.A. Zachariasse for providing his nanosecond TCSPC setup. Finally, we thank M. Takata (Mitsubishi Paper Mills Inc.) and M. Yohei (Inabata UK Ltd.) for discussions on indoline dyes.

\section{Notes and references}

1 T. Horiuchi, H. Miura and S. Uchida, Chem. Commun., 2003, 3036.

2 T. Horiuchi, H. Miura, K. Sumioka and S. Uchida, J. Am. Chem. Soc., 2004, 126, 12218

3 S. Ito, S. M. Zakeeruddin, R. Humphry-Baker, P. Liska, R. Charvet, P. Comte, M. K. Nazeeruddin, P. Péchy, M. Takata, H. Miura, S. Uchida and M. Grätzel, Adv. Mater., 2006, 18, 1202.
4 D. Kuang, S. Uchida, R. Humphry-Baker, S. M. Zakeeruddin and M. Grätzel, Angew. Chem., Int. Ed., 2008, 47, 1923.

5 D. Kuang, J. Brillet, P. Chen, M. Takata, S. Uchida, H. Miura, K. Sumioka, S. M. Zakeeruddin and M. Grätzel, ACS Nano, 2008, 2, 1113 .

6 S. Ito, H. Miura, S. Uchida, M. Takata, K. Sumioka, P. Liska, P. Comte, P. Péchy and M. Grätzel, Chem. Commun., 2008, 5194.

7 W. H. Howie, F. Claeyssens, H. Miura and L. M. Peter, J. Am. Chem. Soc., 2008, 130, 1367.

8 T. Dentani, Y. Kubota, K. Funabiki, J. Jin, T. Yoshida, H. Minoura, H. Miura and M. Matsui, New J. Chem., 2009, 33, 93.

9 M. Matsui, A. Ito, M. Kotani, Y. Kubota, K. Funabiki, J. Jin, T. Yoshida, H. Minoura and H. Miura, Dyes Pigm., 2009, 80, 233.

10 N. O. V. Plank, I. Howard, A. Rao, M. W. B. Wilson, C. Ducati, R. S. Mane, J. S. Bendall, R. R. M. Louca, N. C. Greenham, H. Miura, R. H. Friend, H. J. Snaith and M. E. Welland, J. Phys. Chem. C, 2009, 113, 18515.

11 T. Yoshida, J. Zhang, D. Komatsu, S. Sawatani, H. Minoura, T. Pauporté, D. Lincot, T. Oekermann, D. Schlettwein, H. Tada, D. Wöhrle, K. Funabiki, M. Matsui, H. Miura and H. Yanagi, Adv. Funct. Mater., 2009, 19, 17.

12 U. M. Tefashe, T. Loewenstein, H. Miura, D. Schlettwein and G. Wittstock, J. Electroanal. Chem., 2010, 650, 24.

13 Y. Selk, M. Minnermann, T. Oekermann, M. Wark and J. Caro, J. Appl. Electrochem., 2011, 41, 445.

14 M. Fakis, E. Stathatos, G. Tsigaridas, V. Giannetas and P. Persephonis, J. Phys. Chem. C, 2011, 115, 13429.

15 R. Jose, A. Kumar, V. Thavasi, K. Fujihara, S. Uchida and S. Ramakrishna, Appl. Phys. Lett., 2008, 93, 023125.

16 T. Le Bahers, T. Pauporté, G. Scalmani, C. Adamo and I. Ciofini, Phys. Chem. Chem. Phys., 2009, 11, 11276.

17 A. L. Dobryakov, S. A. Kovalenko, A. Weigel, J. L. Pérez Lustres, J. Lange, A. Müller and N. P. Ernsting, Rev. Sci. Instrum., 2010, 81, 113106.

18 T. Lenzer, S. Schubert, F. Ehlers, P. W. Lohse, M. Scholz and K. Oum, Arch. Biochem. Biophys., 2009, 483, 213.

19 K. Oum, P. W. Lohse, F. Ehlers, M. Scholz, M. Kopczynski and T. Lenzer, Angew. Chem., Int. Ed., 2010, 49, 2230.

20 K. Golibrzuch, F. Ehlers, M. Scholz, R. Oswald, T. Lenzer, K. Oum, H. Kim and S. Koo, Phys. Chem. Chem. Phys., 2011, 13, 6340 .

21 J. Piel, M. Beutter and E. Riedle, Opt. Lett., 2000, 25, 180.

22 E. Riedle, M. Beutter, S. Lochbrunner, J. Piel, S. Schenkl, S. Spörlein and W. Zinth, Appl. Phys. B: Lasers Opt., 2000, 71, 457.

23 M. Kopczynski, T. Lenzer, K. Oum, J. Seehusen, M. T. Seidel and V. G. Ushakov, Phys. Chem. Chem. Phys., 2005, 7, 2793.

24 M. Kopczynski, F. Ehlers, T. Lenzer and K. Oum, J. Phys. Chem. $A, 2007,111,5370$.

25 U. Leinhos, W. Kühnle and K. A. Zachariasse, J. Phys. Chem., 1991, 95, 2013.

26 K. A. Zachariasse, G. Duveneck and R. Busse, J. Am. Chem. Soc., 1984, 106, 1045.

27 Handbook of Chemistry and Physics, CRC Press, Boca Raton, 85 edn, 2004.

28 M. L. Horng, J. A. Gardecki, A. Papazyan and M. Maroncelli, J. Phys. Chem., 1995, 99, 17311.

29 D. Schwarzer, J. Troe, M. Votsmeier and M. Zerezke, J. Chem. Phys., 1996, 105, 3121.

30 T. Lenzer, F. Ehlers, M. Scholz, R. Oswald and K. Oum, Phys. Chem. Chem. Phys., 2010, 12, 8832.

31 A. L. Dobryakov, S. A. Kovalenko and N. P. Ernsting, J. Chem. Phys., 2005, 123, 044502.

32 E. Krystkowiak, K. Dobek and A. Maciejewski, J. Photochem. Photobiol., A, 2006, 184, 250.

33 M. Sajadi, T. Obernhuber, S. A. Kovalenko, M. Mosquera, B. Dick and N. P. Ernsting, J. Phys. Chem. A, 2009, 113, 44.

34 S. J. Strickler and R. A. Berg, J. Chem. Phys., 1962, 37, 814.

35 C. Wohlfarth and B. Wohlfarth, Landolt-Börnstein - Vol. III/38, Optical Constants, Springer, Berlin, Heidelberg, 1996.

36 M. O. Lenz and J. Wachtveitl, J. Phys. Chem. C, 2008, 112, 11973. 